\title{
C-Section on patient with secondary paraplegia resulting from spontaneous spinal epidural hematoma and acquired FXIII deficiency. A case report
}

Kampel M.A., Figar A., Mc Loughlin S., Bonofiglio F.C.

Hospital Italiano de Buenos Aires, Dept of Anaesthesiology, Buenos Aires, Argentina

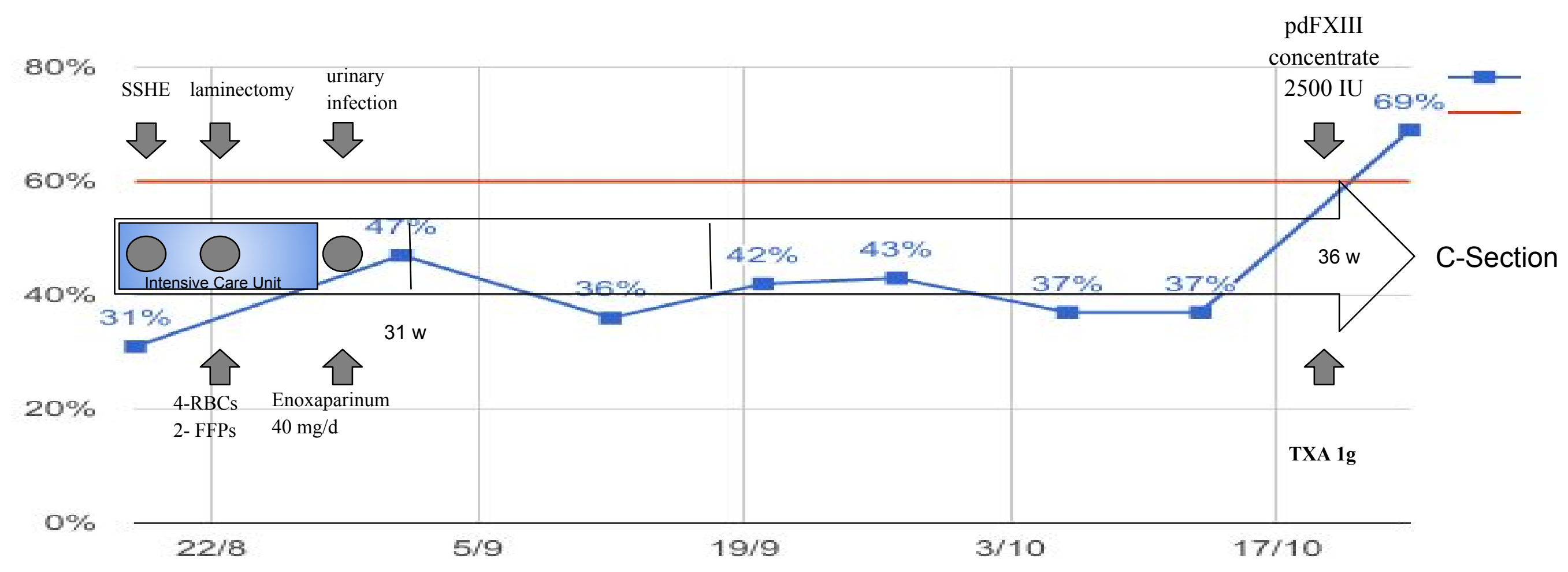

Fig.1: Timeline of events.

Background: Spontaneous spinal epidural hematoma (SSEH) in a pregnant patient is an extremely rare clinical condition that may require emergency surgical evacuation, while termination of pregnancy depends on fetal viability status ${ }^{1}$. Etiologies include: hemorrhagic diathesis, autoimmune inflammatory vasculitis, anticoagulant therapy, vascular malformations, and tumors. Pregnancy is considered a risk factor for SSEH. We present a patient with SSEH and acquired FXIII deficiency (FXIII D) as a sole finding.

Case report: A 37 year old, gravida 3 para 2, 28 weeks of gestation was transferred to our hospital with symptoms of progressive paraplegia over 72 hours. MRI showed spinal lesion due to SSEH and emergency T1--T5 laminectomy was performed. Acute bleeding during laminectomy required transfusions of 4 RBCs and 2 FFPs. Neither FXIII nor Tranexamic acid (TXA) was administered on that occasion. Postoperative neurological examination showed flaccid paraplegia, with sensitive level at T3. Laboratory test results showed normal values except for FXIII-A concentration 28\% (60--160\%). No other major bleeding cause could be determined aside from the FXIII D. Two months later, an elective c--section was performed at 36 weeks of gestation. Plasma- derived FXIII concentrate (pdFXIII) 2500 IU was administered preoperatively along with TXA $1 \mathrm{~g}$. General anesthesia using propofol for induction and sevorane for maintenance was administered, and no muscle relaxants or opioids were used. Videolaryngoscopy was used for orotracheal intubation. Bleeding was between normal ranges. There was no need for RBC transfusions. A healthy female baby was born with a 8/9 Apgar score and 2,840 grams. Post-operative FXIII-A level was $69 \%$.
Discussion: There are merely a few dozens of pregnant SSEH cases reported in the literature. Regional anesthesia for paraplegic condition is advised by some authors as the best technique to prevent autonomic dysreflexia; although general anesthesia seems more appropriate to prevent further spinal complications in patients with hemorrhagic diathesis ${ }^{2}$.

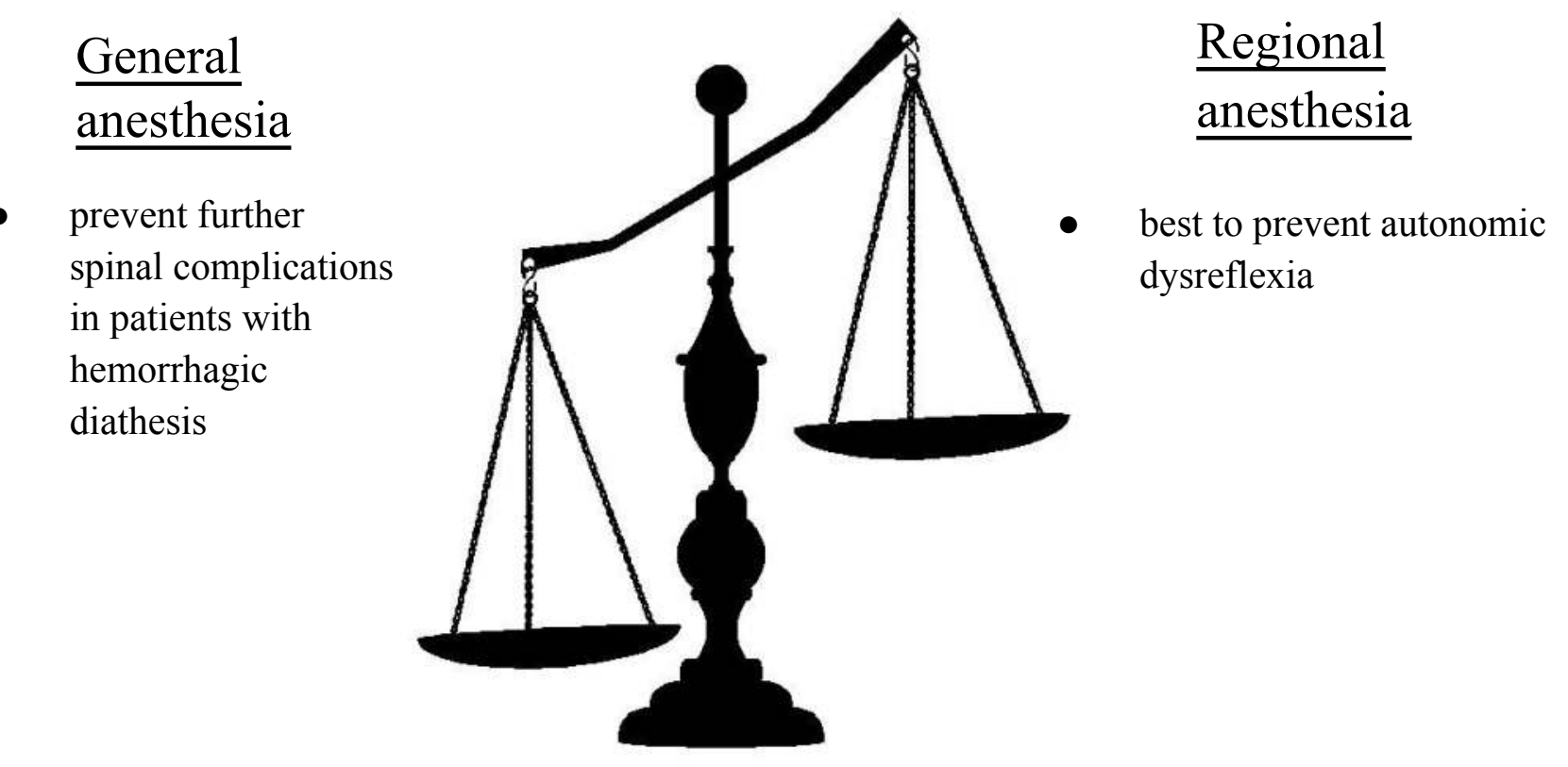

SSEH + Paraplegia + FXIII D + Pregnancy

Fig. 2: Choice of anesthesia technique.

Learning points: General anesthesia is an adequate anesthesia technique for paraplegic patients with FXIIID. FXIII should be tested even if coagulation test results are normal, and preoperative pdFXIII as well as TXA administration can prevent severe bleeding in these patients.

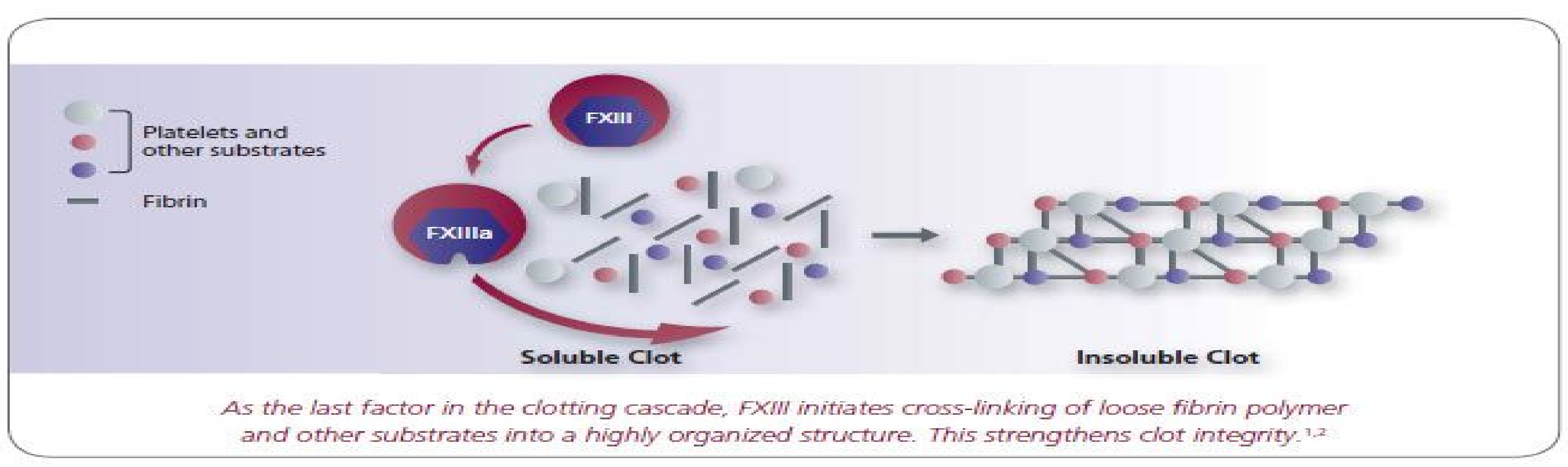

Fig. 3: FXIII function in the clotting cascade. 